\title{
Enhanced Resource-Dependent Critical Path Method for Identifying Resource Dependencies in Variable Resource Constrained Project
}

\author{
Salman Ali NISAR ${ }^{1}$, Koshi YAMAMOTO ${ }^{2}$, Koji SUZUKI $^{3}$ \\ ${ }^{1}$ Doctoral Student, Dept. of Civil Eng., Nagoya Institute of Technology \\ (Gokiso-cho, Showa-ku, Nagoya 466-8555, Japan) \\ E-mail: ali_nisar02@yahoo.co.jp \\ ${ }^{2}$ Professor Emeritus, Dept. of Civil Eng., Nagoya Institute of Technology \\ (Gokiso-cho, Showa-ku, Nagoya 466-8555, Japan) \\ E-mail: yamamoto.koshi@ nitech.ac.jp \\ ${ }^{3}$ Associate Professor, Dept. of Civil Eng., Nagoya Institute of Technology \\ (Gokiso-cho, Showa-ku, Nagoya 466-8555, Japan) \\ E-mail: suzuki.koji@nitech.ac.jp
}

This paper proposes the enhanced resource-dependent critical path method (enhanced RDCPM) for identifying resource dependencies that exist between activities in order to compute the correct floats and determine the critical path in the project schedule under variable resource constraints. In addition to technological relations, there exist resource dependencies between activities in a project schedule under resource constraints that are neglected in traditional resources constrained scheduling (RCS) techniques. Therefore RCS cannot compute the correct floats and critical path(s). Although previous studies have proposed many methods in order to overcome these shortcomings, they assumed that the maximum amount of available resources remains constant during the execution of the project. However, an actual project can have variable resource constraints in certain situations. The enhanced RDCPM, which is an improved version of the RDCPM, reasonably identifies the resource dependencies between activities and computes the correct floats and critical path in a project schedule under either variable or constant resource constraints. Moreover, it provides an updateable schedule with stable work sequences. Therefore, the enhanced RDCPM can be adapted for scheduling and control in real construction projects.

Key words: Construction project schedule, Critical path method, Resource dependent relation, Variable resource constraints.

\section{Introduction}

The scheduling for the construction projects is normally performed using the critical path method (CPM). The CPM, which was developed in the 1950s, provides useful information about a project schedule. Once the project network is formulated the following steps are performed: (i) a forward pass to determine the earliest start time (ES) and earliest finish time (EF) for activities, (ii) a backward pass to determine the latest start time (LS) and latest finish time (LF) for activities, (iii) float calculations and (iv) identification of critical activities and critical path(s). This information is very important for the project manager to plan and control the project more actively and efficiently ${ }^{1)}$.

However, CPM cannot produce a realistic and practical schedule. Because it assumes that there are unlimited resources during the project execution, while some resources are highly limited in real projects $^{2)}$.However, resource constrained scheduling (RCS) techniques can be considered to address resource limitations; they are unable to provide the correct floats and determine the critical path(s). In traditional RCS techniques, only the technological precedence relations between activities are considered in computing total float (TF). However, there are resource dependency relations between activities in a resource constrained project that are ignored by the backward pass of the CPM in the RCS technique $^{2), 3), 4), 5), 6}$. Also, project management software such as Oracle Primavera P6 and MS Project cannot provide the correct float and critical path ${ }^{2)}$.

Several studies have proposed heuristic algorithms to solve the RCS technique limitations. Kim and Garza $^{2)}$, Bowers ${ }^{3)}$, Weist ${ }^{4)}$, Woodworth and Sha- 
nahan ${ }^{5)}$, and $\mathrm{Lu}$ and $\mathrm{Li}^{7)}$ studied project scheduling methods for identify the correct float and critical path(s) in the project schedule under resource constraints. These studies provide useful information for the present problem; however, each method has significant shortcomings ${ }^{8)}$.

To overcome these drawbacks, the authors have proposed a 4-step resource-dependent critical path method $(\mathrm{RDCPM})^{8}$. Although the RDCPM identifies reasonable resource dependencies between activities, and successfully computes the correct floats and critical path, this approach, including all previous literatures, assumes that the maximum amount of available resources remains constant during the execution of the project. However, the maximum amount of resources may vary in different stages of a real project.

Therefore, the authors have developed a scheduling model to identify the critical path and real TF under variable resource constraints ${ }^{9}$. Although this model can successfully provide schedules that accomplish its own objectives, it does not identify the resource dependencies that exist between activities. A schedule under resource constraints, without considering resource dependencies, cannot be updated, and the work sequence is not stable.

For the above reasons, we improved the $\mathrm{RDCPM}^{8)}$ as an enhanced RDCPM to identify resource dependencies between activities in order to compute the real float and critical path in a project schedule under variable resource availability. However, this study assumed that the project is under a single resource constraint and there exist only finish-start technological precedence relationships between activities with zero time lags. Therefore, further research is required to improve this study while considering the multiple resources constraint and the precedence diagram method (PDM) relationships, i.e., finishstart, start-start, start-finish and finish-finish, between activities with positive and negative time lags.

\section{Model formulation and basic assumption}

We consider a single project that consists of activity $j$ (or job $j$ ) $(j=1,2 \ldots, J$ ) with a non-preemptable duration of $d_{j}$ units of time. The activities are linked by two kinds of constraints, namely technological precedence constraints that prevent an activity $j$ from being started before all its predecessors $i$ in a set $P_{j}$ are finished, and with a resource constraint that arose as follows. In order to be processed, activity $j$ requires $k_{j}$ resource units during every period of its occurrence. Because available resources have a limited number of $K_{\rho i}$ units for each period, activity may not be scheduled
F3 (Civil Engineering Informatics), Vol. 69, No. 2, I_110-I_120, 2013.

at its earliest (precedence feasible) start time but later. The project is constrained by one renewable resource $r$, which is variable during different scheduling stages. There exist only finish-start technological precedence relations with zero time lags. The scheduling unit is on a daily basis, and the start time of the project is considered as day 0 . For each activity, the duration, resource requests, and precedence relations are assumed to be deterministic and known in advance. A fenced bar chart is used to represent the project schedule and the activities' relation.

The schedule is divided into $n_{r}$ periods based on the variety of the maximum amount of available resources $r$, i.e. $\rho_{i}=\rho_{1}, \rho_{2} \ldots, \rho_{n r}$. The maximum available resources during the period of $\rho_{i}$ is $K_{\rho i}$ units, where $K_{\rho i} \neq K_{\rho i+1}, \forall i \in\left\{1,2 \ldots, n_{r}-1\right)$. In order to make the maximum available resource constant throughout the schedule period, $n_{r}$ number of dummy activities, $\beta_{i}=\beta_{1}, \beta_{2} \ldots, \beta_{n r}$, are inserted into the schedule in which $\beta_{i-1}$ preceded $\beta_{i}$. The duration of dummy activity $\beta_{i}$ is $d_{\beta i}=\rho_{i}$. Let $E S_{\beta 1}=L S_{\beta 1}=0 ; E F_{\beta i}$ $=L F_{\beta i}=\left(E S_{\beta i}\right.$ or $\left.L S_{\beta i}\right)+d_{\beta i}$, for $\forall i \in\left(1,2 \ldots, n_{r}-1\right)$; $E S_{\beta i}=L S_{\beta i}=E F_{\beta i-1}=L F_{\beta i-1}$, for $\forall i \in\left(2,3 \ldots, n_{r}\right)$; and $E F_{\beta n r}=L F_{\beta n r}=T_{r}$, where $E S_{\beta i}, L S_{\beta i}, E F_{\beta i}, L F_{\beta i}$, and $d_{\beta i}$ are the earliest start time, latest start time, earliest finish time, latest finish time, and the duration of dummy activity $\beta_{i}$, respectively. $T_{r}$ is the shortest project duration under resource constraints. It is assumed that each dummy activity $\beta_{i}$ requires $k_{\beta i}$ units of artificial resources: $k_{\beta i}=\operatorname{Max}\left(K_{\rho 1}, K_{\rho 2} \ldots\right.$, $\left.K_{\rho n r}\right)-K_{\rho i}$

\section{Enhanced RDCPM}

The enhanced RDCPM, which is an improved version of the $\mathrm{RDCPM}^{8)}$ approach, is developed as a heuristic method based on the traditional CPM and RCS techniques. The concept of the enhanced RDCPM is composed of 6 steps. To better demonstrate the method, each step is described along with a simple project schedule which is the construction of a partial of a warehouse. The project consists of: providing concrete floor of the warehouse, a pavement roadway, and walkways of the site. The warehouse floor is divided in two areas. Area 1 needs for steel reinforcement (rebar) concrete flooring and area 2 needs to be prepared by plain concrete (non-steel reinforcement concrete) flooring. This small project is broken into 6 activities (jobs). Table 1 shows the activity name, the activity description, the logical relationship (Precedence relationship), the duration, and the required number of labor (resource). Activities SS and EE are assumed to be artificial activities indicating the project commencement and the project 
Table 1 Activity data of simple schedule

\begin{tabular}{cclccc}
\hline \multicolumn{2}{l}{ Priority Activity } & \multicolumn{1}{c}{ Activity Description } & D & R & Pr \\
\hline- & SS & Start of Project & 0 & 0 & - \\
2 & A & Provide Pavement Roadway & 4 & 4 & SS \\
1 & B & Instal Formwork and Reinforcement & 5 & 3 & SS \\
& & Arangement for Area 1 & & & \\
3 & C & Instal Formwork for Area 2 & 3 & 1 & SS \\
6 & D & Provide Walkways & 3 & 1 & SS \\
4 & E & Concrete Placement for Area 1 & 4 & 2 & B \\
5 & F & Concrete Placement for Area 2 & 4 & 2 & C \\
- & EE & End of Project & 0 & 0 & A, D, E, F \\
\hline * D=Duration & R=Resource Pr=Predecessor Activity & & &
\end{tabular}

completion, respectively. Their durations and resource requirement are zero.

As shown the logical schedule network in Fig.1, once the project starts, activities A, B, C, and D can be started. However, activity E cannot be started until the activity B has been finished. In other words, we cannot place the concrete until the formwork and steel reinforcement are erected. As well as activity $\mathrm{F}$ cannot be started before the completion of activity $\mathrm{C}$. Note that concrete operations of each area need two more activities, i.e., concrete curing and de-mold the formwork. However, they are ignored for the sake of simplicity. In this small project, we assume that the maximum available labors per each day are limited, and vary during the execution of the project as follows. For the first 4 days, there are 4 labors, for days $5-9$, there are 7 labors, and after day 9 to the end of the schedule, there are 5 labors.

\section{(1) Step 1: Performing CPM}

Assuming unlimited resources, traditional CPM forward and backward passes ${ }^{1), 12}$ are employed to find the $E S, L S, E F, L F$, and floats for each activity. The result of the CPM for the simple schedule is shown in Fig.2, for which the TF and critical path (the sequence activities denoted by the bold line) are identified. However, if the maximum available resources (labors) are considered, resource required on days 1-4 are over the limit. Therefore, the RCS technique is required to set the given resource limits.

\section{(2) Step 2: Inserting dummy activities}

If the amount of available resources varies during the execution of a project, it would increases the difficulty with which the schedule is provided; moreover, it may not be possible to identify resource

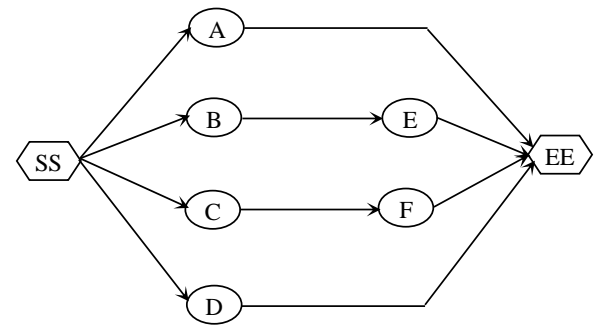

Fig.1 Simple schedule network
Journal of Japan Society of Civil Engineers, Ser.

F3 (Civil Engineering Informatics), Vol. 69, No. 2, I_110-I_120, 2013.

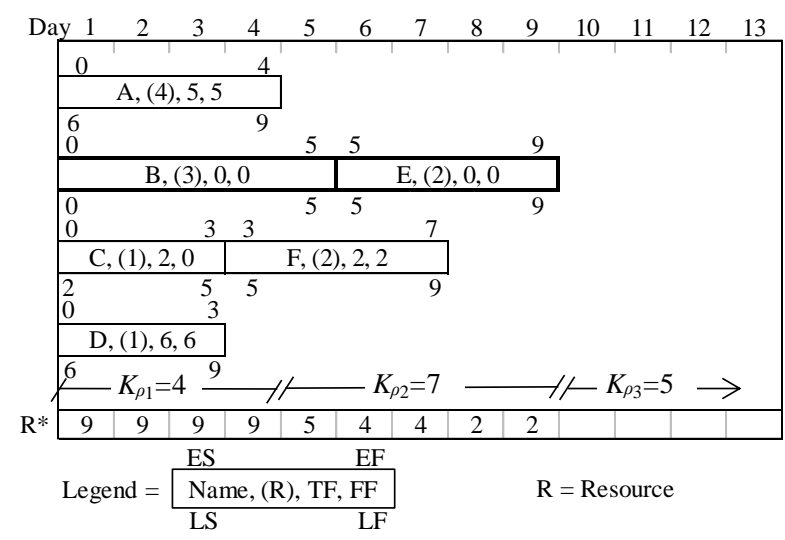

Fig.2 CPM schedule on Fenced Barnhart in Step 1

dependencies for some activities. This step aims to maintain the maximum available resource constant during the scheduling processes by inserting dummy activities into the schedule. Therefore, the schedule is divided into several periods based on the availability of resource along the schedule. The dummy activity is inserted into each period afterwards.

For instance, in Fig.2, the maximum amount of available resources varies in three stages, i.e., $K_{\rho 1}=4$, $K_{\rho 2}=7$, and $K_{\rho 3}=5$, along the simple schedule. Thus, the schedule is partitioned into three periods. The duration of each period is given by $\rho_{1}=4, \rho_{2}=5$, and $\rho_{3}=T_{r}-9$, respectively. As shown in Fig.3, three dummy activities, i.e., $\beta_{1}, \beta_{2}$, and $\beta_{3}$, are inserted into the schedule. The duration and the artificial resource for each dummy activity are determined as: $d_{\beta 1}=4$, $d_{\beta 2}=5, d_{\beta 3}=T_{r}-9$ and $k_{\beta 1}=K_{\rho 2}-K_{\rho 1}=7-4=3, k_{\beta 2}$ $=K_{\rho 2}-K_{\rho 2}=7-7=0, k_{\beta 3}=K_{\rho 2}-K_{\rho 3}=7-5=2$, respectively. As shown in Fig.3, the maximum available resources, including the artificial resources of the dummy activities, then become constant along the schedule, i.e. $K_{r}=7$. The RCS technique can now be easily applied on the schedule.

Moreover, the dummy activity acts as an important element in the project schedule under variable resource constraints. If a resource(s) is transferred from a dummy activity to a specific activity or vice versa, then a resource link is required between them. Therefore, dummy activities can help to establish

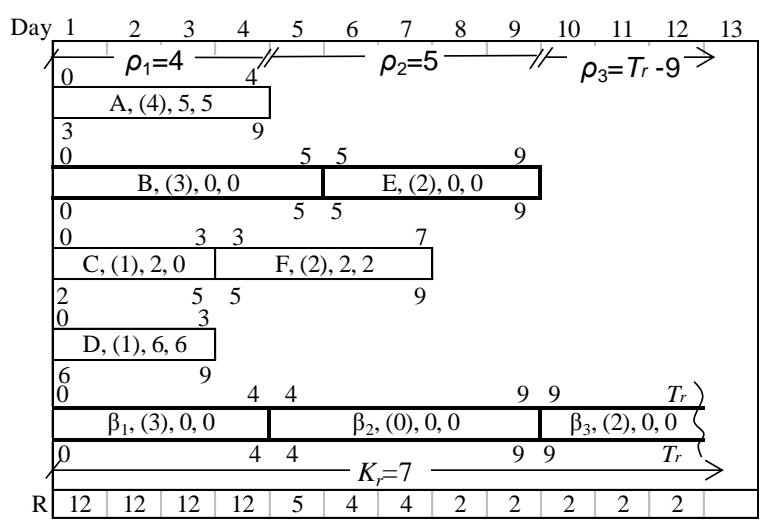

Fig.3 CPM schedule with dummy activities in Step 2 
resource links between activities so that they provide an updateable schedule with stable work sequences. Let us to evaluate the efficiency of the dummy activity in a simple schedule. As shown in Fig.4 (a), the maximum available resource is 3 units on days 1,2 , and 3 , and is increased to 5 units after day 3 . The ES of activity $\mathrm{A}$ is determined by the RCS technique as 3 , because there is a sufficient resource for it $\left(\mathrm{Kr}_{2}=\right.$ 5). Therefore, there is no resource transfer between a specific activity to activity A; hence, no resource link is required. In this situation, the activity $\mathrm{A}$ is not included in a stable work sequence, and the schedule therefore cannot be updated.

However, as shown in Fig.4 (b), establishing a resource link from activity $B$ to activity A with negative lag (FS $=-1)$ may provide a stable work sequence for activity A, but it cannot generate a reasonable schedule. For instance, as shown in Fig.4 (b), after establishing the resource link, the 1-day TF of activity $\mathrm{B}$ is eliminated. Moreover, if the duration of activity $B$ is extended or its finish time is postponed, it will delay the ES of activity A. Meanwhile, sufficient resources are available for both activities A and B after day 3 of the project. Hence, any dally of activity B should not affect the ES activity A.

Alternatively, as shown in Fig.4 (c), after inserting dummy activities, i.e., $\beta_{1}$ and $\beta_{2}$, and allocating the artificial resource for dummy activities, the maximum amount of available resources became constant during scheduling. Resources (two units) are transferred from dummy activity $\beta_{1}$ to activity A; hence, a resource link is established between them. Therefore, by inserting dummy activities, the enhanced RDCPM will provide a reasonable and updatable schedule with stable job sequences. Note that the artificial resources which are allocated to dummy activities consume zero cost. Therefore, the project team should take care to the amount of artificial resources that are allocated in the schedule in order to they should not affect the cost of project.

\section{(3) Step 3: RCS forward pass calculation}

In this step, based on the results of Step 1 and 2, we employed the RCS forward pass calculation, which is similar to the serial heuristic of the RCS
F3 (Civil Engineering Informatics), Vol. 69, No. 2, I_110-I_120, 2013.

technique, to set the maximum available resources and to omit the resources that are overloaded by delaying the $E S_{j}$ of the activities. The processes consist of at most $g\left(g=1,2 \ldots, J+n_{r}\right)$ stages, in each of which has one activity that is selected, and its required resource is allocated, after which the activity is scheduled. There is a schedule time $t_{g}$ and three activity-sets in each stage. Activities that are completed up to $t_{g}$ are placed in the completed set $C_{g}$. Activities which are already scheduled, but which remain active during the schedule time, are in the active set $A_{g}$. Finally, activities that are eligible to be scheduled, i.e., those activities for which the predecessors are already completed, are in the eligible set $E_{g}$. Each stage consists of two steps. (a) The new schedule time $t_{g}$ is determined, and it is nominated as the EF of activity(s) that is in the active set, and finished on the new schedule time. Afterward, the activity(s) is removed from the active set and put into the complete set. In this step, some activity(s) may become eligible to be scheduled, and is/are placed into the eligible set since all of its predecessor activities are scheduled. (b) One or more eligible activity(s) $j_{g}$ that has the highest priority with considering available resource feasibility in $K_{r}$, is selected from set $E_{g}$, and its required resource is allocated, after which the activity is scheduled. The priority is determined based on the characteristics of activities such as the least TF, the least LS, the largest duration, and so on. The schedule time $t_{g}$ is determined as the ES of the selected activity. Afterwards, the selected activity is moved from the eligible set to the active set. Because the ES of dummy activity $\beta_{i}$ is fixed and cannot be delayed, it has the highest priority to be scheduled and its required resource(s) is allocated in stage $t_{g}=E S_{\beta i}$. If there is not enough resource in $K_{r}$ for dummy activity $\beta_{i}$, one or more activities will be returned from the active set to the eligible set so that their resources are allocated to the dummy activity. This process is repeated until all activities are scheduled and placed in the complete set.

Table 2 lists the processes of the RCS forward pass for the simple schedule at each stage. In the simple schedule in this study, the priority is determined by the rank position weight method ${ }^{11)}$. Col-

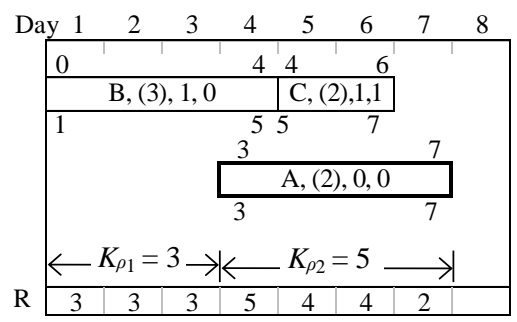

(a) RCS schedule without resource link

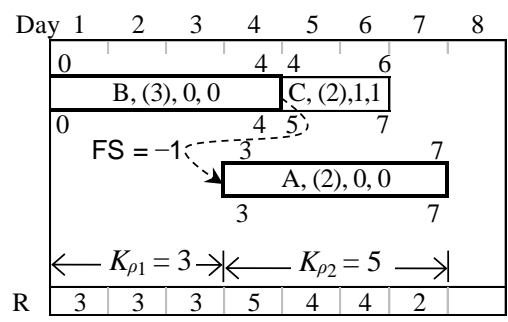

(b) RCS schedule with resource link and negative lag

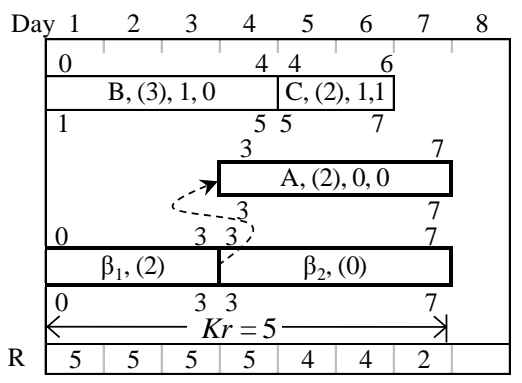

(c) Enhanced RDCPM schedule

Fig.4 Evaluation of the efficiency of dummy activities in a schedule under variable resource constraints 
Table 2 RCS forward pass process on simple schedule

\begin{tabular}{|c|c|c|c|c|c|}
\hline$g$ & $t_{g}$ & $E_{g}$ & $A_{g}$ & $C_{g}$ & $j_{g}$ \\
\hline 1 & 0 & $\left\{\mathrm{~A}, \mathrm{~B}, \mathrm{C}, \mathrm{D}, \beta_{1}\right\}$ & \{\} & \{\} & $\left\{\beta_{1}, \mathrm{~B}, \mathrm{C}\right\}$ \\
2 & 3 & $\{\mathrm{~A}, \mathrm{D}, \mathrm{F}\}$ & $\left\{\mathrm{B}, \beta_{1}\right\}$ & $\{\mathrm{C}\}$ & $\{\mathrm{D}\}$ \\
3 & 4 & $\left\{\mathrm{~A}, \mathrm{~F}, \beta_{2}\right\}$ & $\{\mathrm{B}, \mathrm{D}\}$ & $\left\{\mathrm{C}, \beta_{1}\right\}$ & $\left\{\beta_{2}, \mathrm{~F}\right\}$ \\
4 & 5 & $\{\mathrm{~A}, \mathrm{E}\}$ & $\left\{\mathrm{D}, \mathrm{F}, \beta_{2}\right\}$ & $\left\{\mathrm{C}, \mathrm{B}, \beta_{1}\right\}$ & $\{\mathrm{A}\}$ \\
5 & 6 & $\{\mathrm{E}\}$ & $\left\{\mathrm{A}, \mathrm{F}, \beta_{2}\right\}$ & $\left\{\mathrm{C}, \mathrm{B}, \mathrm{D}, \beta_{1}\right\}$ & \{\} \\
6 & 8 & $\{\mathrm{E}\}$ & $\left\{\mathrm{A}, \beta_{2}\right\}$ & $\left\{\mathrm{C}, \mathrm{B}, \mathrm{D}, \mathrm{F}, \beta_{1}\right\}$ & $\{\mathrm{E}\}$ \\
7 & 9 & $\left\{\beta_{3}\right\}$ & $\{\mathrm{E}\}$ & $\left\{\beta_{2}, \mathrm{~A}, \mathrm{~B}, \mathrm{C}, \mathrm{D}, \mathrm{E}, \mathrm{F}, \beta_{1}\right\}$ & $\left\{\beta_{3}\right\}$ \\
8 & 12 & \{\} & \{\} & $\left\{\mathrm{A}, \mathrm{B}, \mathrm{C}, \mathrm{D}, \mathrm{E}, \mathrm{F}, \beta_{1}, \beta_{2}, \beta_{3}\right\}$ & $\left\{\beta_{3}\right\}$ \\
\hline
\end{tabular}

umn 1 in Table 1, shows the rank of the priority for each activity, where a smaller number indicates a higher priority. As shown in Table 2, at stage $g=1$, the schedule time is determined $t_{1}=0$. As shown in Fig.3, activities A, B, C, D, and $\beta_{1}$ are eligible to be scheduled on day 0 , so they are put into the eligible set, i.e., $E_{1}=\left\{\mathrm{A}, \mathrm{B}, \mathrm{C}, \mathrm{D}, \beta_{1}\right\}$. The sets of $A_{1}$ and $C_{1}$ are empty because there are no active or complete activities on day 0 . As shown in column 6 of Table 2, activities $\beta_{1}, \mathrm{~B}$, and $\mathrm{C}$ are selected by the priority order and considering the available resource feasibility; then, the resource is allocated to each selected activity, i.e., $k_{\beta 1}=4, k_{\mathrm{B}}=3$, and $k_{\mathrm{C}}=1$. The process is terminated at stage 8 , where all activities are placed into the completed set. The project duration is determined to be 12 days $\left(t_{8}=T_{r}=12\right)$. As shown in Fig.5, the RCS forward pass set the maximum available resources and determined the $E S_{j}$ and $E F_{j}$ for each activity.

\section{(4) Step 4: RCS backward pass calculation}

The purpose of the RCS backward pass is to find the $L S_{j}$ and $L F_{j}$ for each activity considering the resource constraints.

\section{a) Step 4.1}

The original schedule is reversed as all predecessor activities are changed to successor ones, and vice versa. Only the forward pass of the CPM is employed on the reversed schedule to find the $E S_{j}$ and $E F_{j}$ for each activity.

\section{b) Step 4.2}

Based on the ES and project duration of Step 3 (RCS schedule), the constraint's LF (C-LF) is calculated from Equation 1 and is imposed on each activity in the reverse CPM schedule. The C-LF is

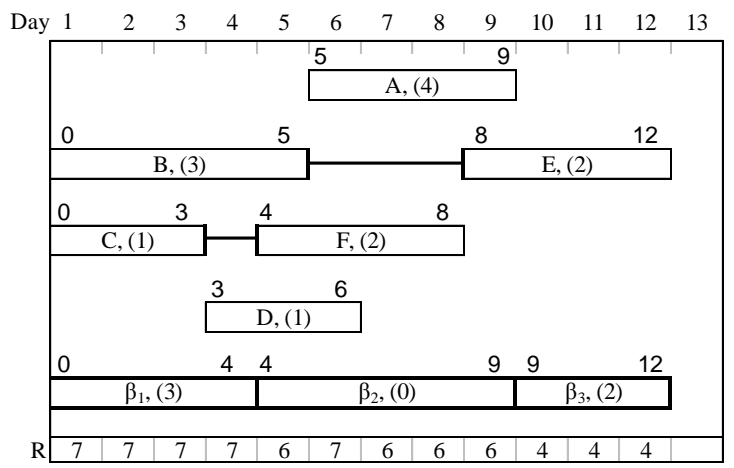

Fig.5 RCS schedule in Step 3 defined as a time restriction imposed on each activity that limits the latest time by which it can be completed.

$$
C-L F_{C R j}=T_{r}-E S_{R j}
$$

where $C-L F_{C R j}$ is the constraint's latest finish time for activity $j$ in the reversed CPM schedule, $T_{r}$ is the minimum project duration in the RCS schedule in Step 3, and $E S_{R i}$ is the earliest start time of activity $j$ in RCS schedule in Step 3.

The $L S_{j}$ and $T F_{j}$ are found by the CPM calculation while considering $C-L F_{C R j}$. Fig.6 shows the reverse CPM schedule with $C-L F_{C R j}$.

\section{c) Step 4.3}

After calculating the $C-L F_{C R j}$ for each activity of reverse CPM schedule, based on the serial heuristic method of the RCS technique, which is similar to RCS forward pass in Step 3, the maximum available resources are set by delaying the activities within their $T F$. The activity is scheduled and resources are allocated by the $C-L F_{C R j}$ in ascending order.

Note that the project duration is fixed and is equal to the result of RCS schedule in Step 3. Therefore, an activity cannot be delayed beyond its TF; otherwise, the project duration will be extended. Because the $C-$ $L F_{C R j}$ for all activities is calculated based on the RCS schedule in Step 3, there is a sufficient number of TFs for activities to set the maximum resource availability. The reverse RCS schedule, which is provided by Step 4.3, is shown in Fig.7.

\section{d) Step 4.4}

Based on the results of Step 4.3 (reverse RCS schedule), the $\mathrm{C}-\mathrm{LF}$ is calculated from Equation 2 and is imposed on each activity in the RCS schedule.

$$
C-L F_{R j}=T_{r}-E S_{R R j}
$$

where $C-L F_{R j}$ is the constraint's latest finish time for activity $j$ in the RCS schedule, and $E S_{R R j}$ is the earliest start time of activity $j$ in the reverse RCS schedule.

According to the CPM calculation and considering

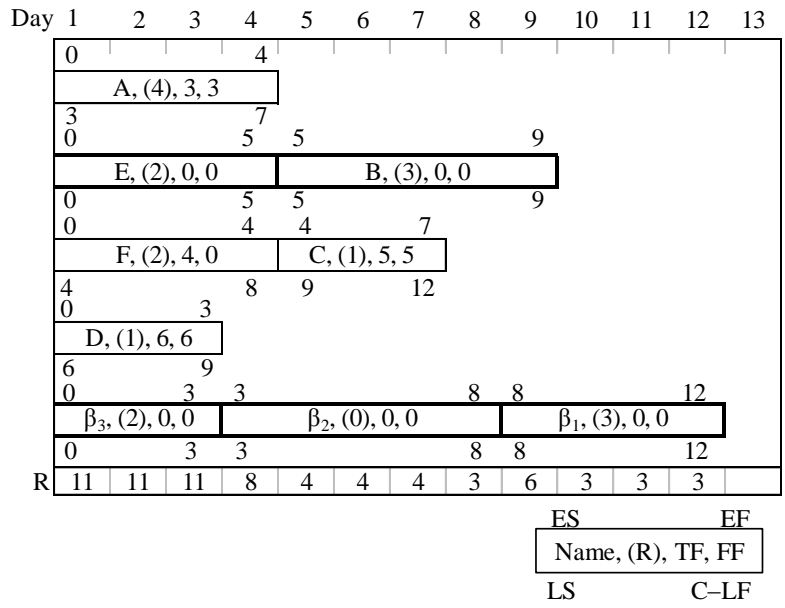

Fig.6 Reverse CPM schedule with C-LF in Step 4.1 and 4.2 


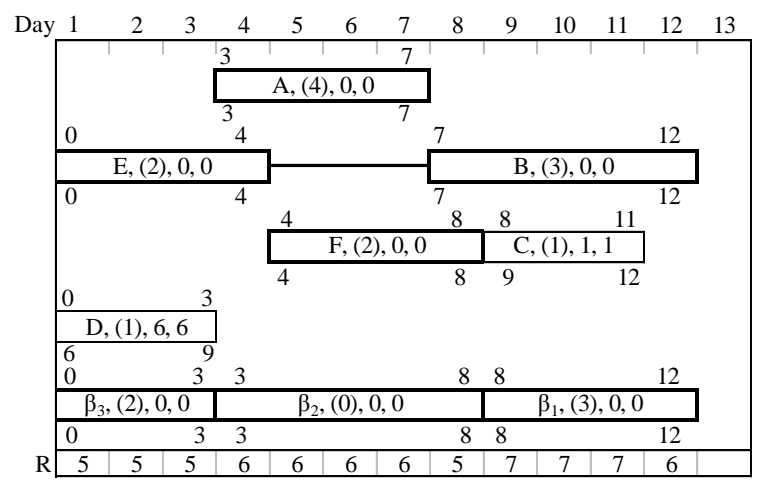

Fig.7 Reverse RCS schedule in Step 4.3

the $C-L F_{R j}$, the $L F_{j}, T F$, and free float $(F F)$ are calculated for each activity of RCS schedule afterwards. Note that the FF will be calculated based only on the technological relation because the resource link is not yet created. Thus, some FFs may be calculated to be greater than the TF for a certain activity. If so, we assume FF $=$ TF because FF cannot be greater than $\mathrm{TF}^{12)}$. As shown by the result of Step 4.4 in Fig.8, the critical path (the sequence activities denoted by the bold line) and most of the correct TFs were identified. However, some TFs may still be incorrect, and resource links are not identified. Therefore, the work sequence, unlike CPM, may not be stable; hence, the schedule cannot be updated.

\section{(5) Step 5: Identifying resource dependency}

As indicated by the authors in the RDCPM ${ }^{8)}$, there exist two types of resource dependency relations: strict resource precedence relations and flexible resource precedence relations, depending on the effect of delaying a predecessor activity $i$ on its current activity $j$ if there is no technological relation between them.

Strict resource precedence relation (SRPR): If the finish time of predecessor activity $i$ is delayed by unit time $a$, the start time of one or more current activity $j$ is immediately delayed by the same unit. Then, the relation between the predecessor activity $i$ and the current activity $j$ is called the SRPR, and the predecessor activity is called a strict resource predecessor activity. This relation is provided during the transfer

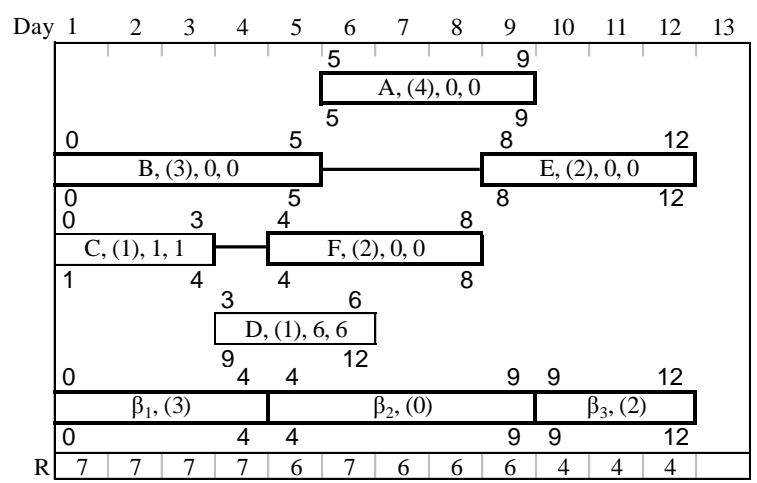

Fig.8 RCS schedule with C-LF in Step 4.4
Journal of Japan Society of Civil Engineers, Ser. F3 (Civil Engineering Informatics), Vol. 69, No. 2, I_110-I_120, 2013.

of resources from the predecessor activity to the current activity by the RCS forward and backward pass calculations.

Flexible resource precedence relation (FRPR): If the finish time of predecessor activity $j$ is delayed by unit time $a$, it will delay the start time of one or more current activities by unit time $a$, where $a>a^{\prime}$. In other words, if the finish time of the predecessor activity is delayed by at least more than one unit time, it affects the start time of the current activity. Then, the relation between the predecessor activity and current activities is called the FRPR, and the predecessor activity is nominated as the flexible resource predecessor activity.

While identifying resource dependencies between activities, the dummy activity is considered as an actual activity. Therefore, in enhanced RDCPM, the SRPR and FRPR, which are similar to the resource dependency in $\mathrm{RDCPM}^{8)}$, are identified in five cases. After identifying the resource relations, the resource link between activities based on a resource link optimization model is established in order to decrease the complexity of the schedule network. A more detailed description of identification of the resource dependency and the optimization of the resource links with examples are provided in $\mathrm{RDCPM}^{8)}$.

As shown in Fig.9, the enhanced RDCPM reasonably identified the resource dependency, and created resource links for the simple schedule under variable resource constraints. Hence, the correct floats and critical path are computed successfully. Fig.10 shows the simple schedule network with resource links that are provided by the enhanced RDCPM. However, after providing resource links, some technological relations may become redundant. These redundant relations do not cause any time calculation errors, but the complexity of the scheduling network will be significantly increased. Therefore, an additional step is required to identify and remove the redundancy.

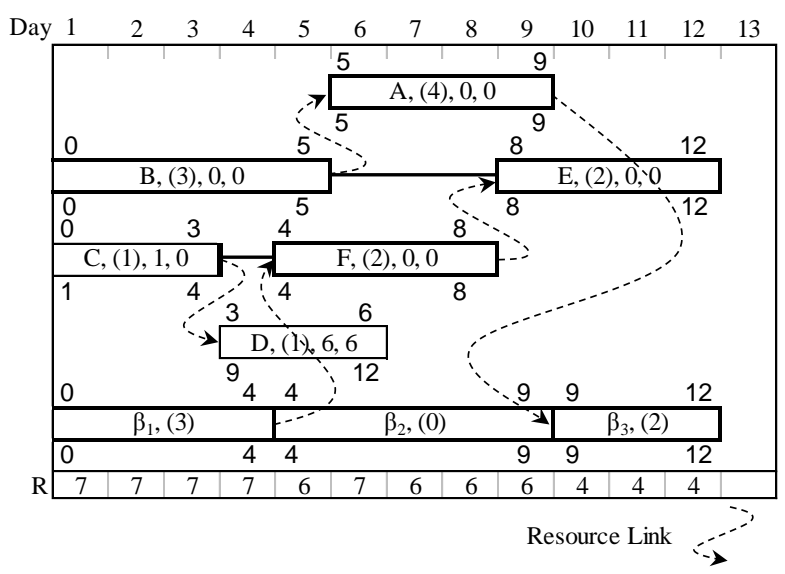

Fig.9 Enhanced RDCPM schedule 


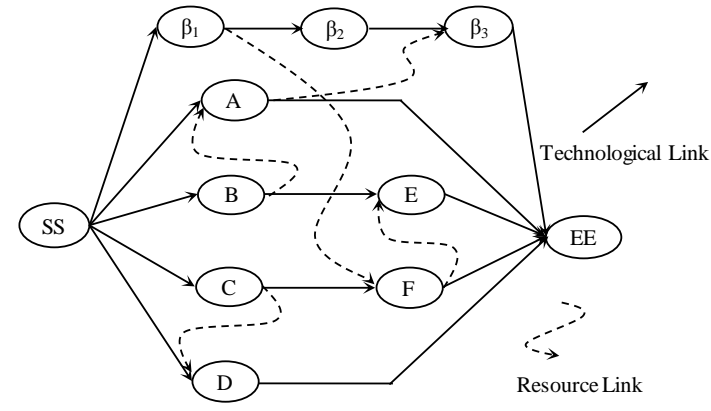

Fig.10 Enhanced RDCPM schedule network in Step 5

\section{(6) Step 6: Removal of redundant precedence relation}

The redundancy is defined as follows: If one activity among the immediate predecessor set of current activities is a predecessor for several other activities in the same set, then the relation between that activity and its current activity is redundant ${ }^{13)}$. For example, in Fig.10, the relation between activities SS and $\mathrm{D}$ is redundant. In addition to both activities, SS and $\mathrm{C}$ are the immediate predecessors of activity $\mathrm{D}$, activity SS preceded the activity C. Hence, this relation can be safely eliminated. The authors introduced a simple method to identify and remove the redundancy in $\mathrm{RDCPM}^{8)}$.

Fig.11 shows the schedule network after removing the redundant relations. This schedule network is now stable and can be updated; moreover, it is applicable to any kind of scheduling method, e.g., CPM and scheduling project management software such as Oracle Primavera P6 and MS Project.

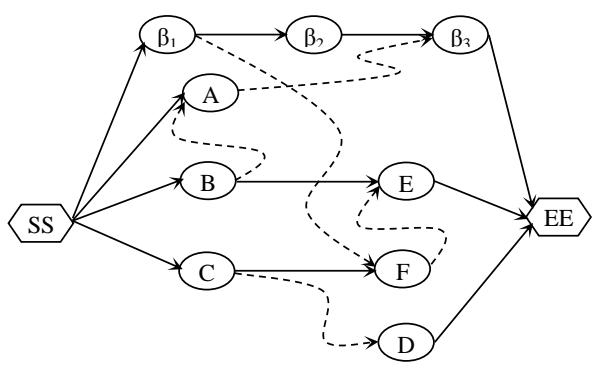

Fig.11 Enhanced RDCPM schedule network after removal of redundant relations

\section{Contribution of enhanced RDCPM to project management software}

Today, the use of project management software is vital in the success of a construction project. Current Windows-based software programs provide a user-friendly working environment. Depending on the sophistication of the software, it can manage estimation and planning, scheduling, cost control and budget management, resource allocation, communication, decision-making, quality management and documentation or administration systems. One of the most common project management software tool types is scheduling tools. Scheduling tools are used to sequence project activities and assign dates and resources to them.

Primavera and MS project are the most widely used in planning, scheduling and controlling of construction project. As a survey was done by Galloway ${ }^{14)}$ in the US, over $64 \%$ of construction companies indicated that they used Primavera software, and over $20 \%$ requiring MS Project. However, these powerful software packages compute all the information about the project schedule based on traditional CPM and RCS technique. Because of the shortages of RCS, they generate incorrect TF, and cannot determine the critical path. For example, the MS Project by RCS technique in Fig.12 determined TFs of activities A, C, D, and F as 3, 4, 6, and 4 days, respectively. However, they would not able to use the determined TFs because of existing of resource constraints. Moreover, the MS Project could not identify the critical path, and it broke the job sequence in simple schedule. For example, activities A and D in Fig.12 are not included in a work sequence. Therefore, this schedule is not stable, and project manager cannot update it. On the other hand, as shown in Fig13, the output data by enhanced RDCPM from Fig.11 is inter into MS Project by which the correct $\mathrm{TF}$, the critical path, and stable work sequence are provided successfully.

Since the concept of RDCPM can solve the aforementioned problems, it may contribute such software packages in order to generate a reasonable schedule for resource-constrained project.

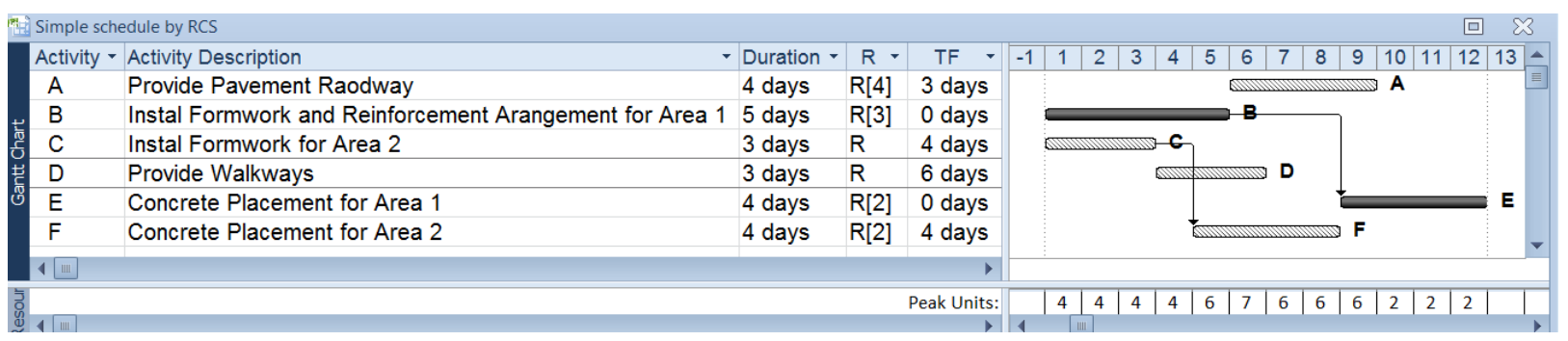

Fig.12 simple schedule output by RCS in MS Project 


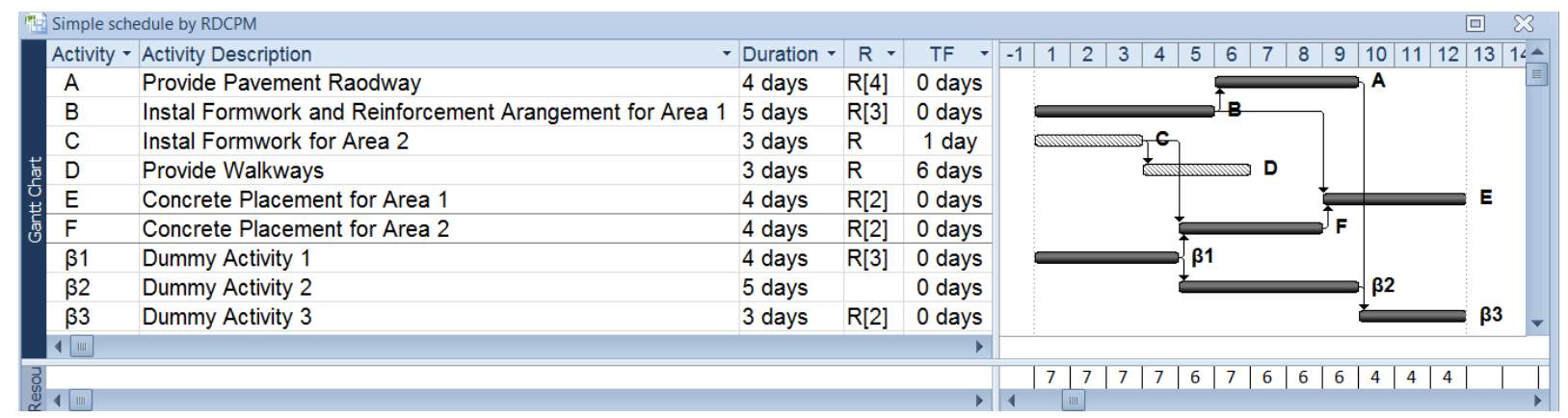

Fig.13 Simple schedule output by RDCPM in MS Project

\section{Case study}

The performance of the enhanced RDCPM is evaluated on a slightly more complicate case study schedule which is taken from the project scheduling problem library (PSPLIB). The library's PSBLIB contains different instance sets for resource constrained project scheduling problems. Researchers use the data sets to evaluate their algorithms. The instances have been generated by the standard project generator (ProGen) ${ }^{15}$. The activity's data for case study schedule is shown in Table $\mathbf{3}$, and the initial schedule network is drawn on Activity-On-Node (AON) diagram in Fig.14. The schedule consists of 32 activities and required a single type of resource. The activities 1 and 32 (source and sink) are artificial activities with zero duration. We supposed to the

Table 3 Schedule data of case study

\begin{tabular}{|c|c|c|c|}
\hline Activity & Duration & Predecessor & Resource \\
\hline 1 & 0 & & 0 \\
\hline 2 & 6 & 1 & 0 \\
\hline 3 & 4 & 1 & 0 \\
\hline 4 & 2 & 1 & 0 \\
\hline 5 & 1 & 3 & 0 \\
\hline 6 & 5 & 5 & 0 \\
\hline 7 & 1 & 6 & 0 \\
\hline 8 & 3 & 3 & 9 \\
\hline 9 & 5 & 7 & 8 \\
\hline 10 & 6 & 8 & 0 \\
\hline 11 & 6 & 2 & 0 \\
\hline 12 & 4 & 2,5 & 0 \\
\hline 13 & 1 & 9 & 0 \\
\hline 14 & 6 & 5 & 1 \\
\hline 15 & 5 & 2 & 0 \\
\hline 16 & 3 & 3 & 0 \\
\hline 17 & 3 & 10,14 & 2 \\
\hline 18 & 2 & 16 & 0 \\
\hline 19 & 3 & 18 & 8 \\
\hline 20 & 3 & 8 & 0 \\
\hline 21 & 7 & 9 & 0 \\
\hline 22 & 4 & 13,19 & 0 \\
\hline 23 & 8 & 13,18 & 0 \\
\hline 24 & 1 & $15,22,23$ & 0 \\
\hline 25 & 8 & $10,11,12$ & 9 \\
\hline 26 & 8 & 17 & 5 \\
\hline 27 & 5 & 4 & 9 \\
\hline 28 & 1 & $11,7,26$ & 4 \\
\hline 29 & 5 & $12,20,24$ & 4 \\
\hline 30 & 2 & $25,27,28$ & 9 \\
\hline 31 & 1 & 13,21 & 0 \\
\hline 32 & 0 & $29,30,31$ & 0 \\
\hline
\end{tabular}

maximum amount available resource varies during the scheduling process as follows. For days 1-15, there are 11 units, for days 16-25, there are 8 units, and for days 26 to the end of the project, there are 11 units. Without considering the given resource constraints, the total duration, determined by CPM calculation, is 31 days.

Fig.15 presents the output by RCS Technique in MS Project for case study schedule. As shown in Fig.15, the RCS technique has successfully leveled the maximum available resources, and has obtained the minimum schedule duration, i.e., 47 days. However, it could not determine all critical activities and the critical path. For example, as shown in Fig.15, only activities 3, 8, 10, 25 and 30 are determined as critical. While, there should be at least one critical path (sequence of critical activities) that shows the longest ordered sequence of activities through the project schedule. Moreover, the RCS technique provided incorrect floats almost for all activities.

Fig.16 presents the case study schedule network including the resource links, after application of the enhanced RDCPM. The enhanced RDCPM reasonably identified resource dependencies between activities, removed the redundant relations, determined the critical path and critical activities, and computed the correct floats. The critical path and critical activities are denoted by bold line in Fig.16. Moreover, this schedule network is now stable and can be updated like CPM schedule. In addition, it is applicable to any kind of scheduling method, e.g., CPM, PERT, and scheduling project management software such as Primavera P6 and MS Project. Once a schedule method such as CPM, is applied to the enhanced RDCPM network, the critical path and the correct floats can be determined by the CPM calculation rules, and the maximum available resource will be satisfied.

The enhanced RDCPM results including resource links and dummy activities are input to MS Project 2010 for comparison the output with traditional RCS technique. As shown in the results of enhanced RDCPM that are output from MS Project, in Fig.17, 


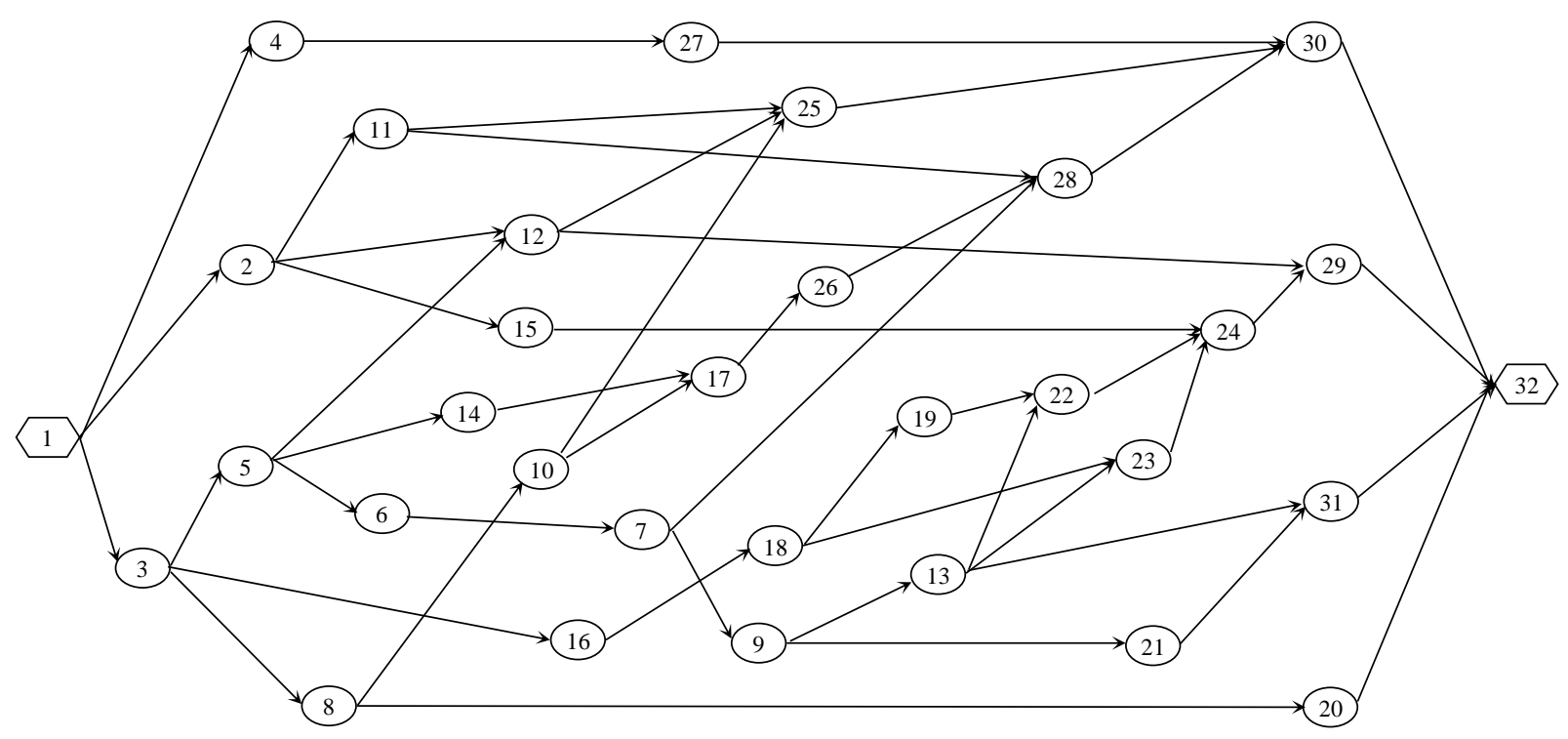

Fig.14 Initial schedule network of case study

the critical path and all the critical activities are determined reasonably, and the floats for each activity are computed correctly.

\section{Conclusion}

Traditional resource constraint scheduling (RCS) techniques cannot provide the correct $\mathrm{TF}$ and the critical path(s). This is because in traditional RCS techniques, only the technological precedence relations between activities are considered when computing TF and FF. However, there are resource-dependent relations between activities in a project schedule under resource constraints that is ignored by CPM backward pass on the RCS technique. Previous studies have focused on the development of methods to solve this problem. However, each method has significant shortcomings.

To overcome these shortcomings, the authors have developed a 4-step heuristic method called the resource-dependent critical path method (RDCPM). Although the RDCPM reasonably identifies the resource dependencies and computes the correct floats and critical path, it assumes that the maximum amount of available resources is constant. However, in some situations, the maximum available resources can be varied during execution of projects.

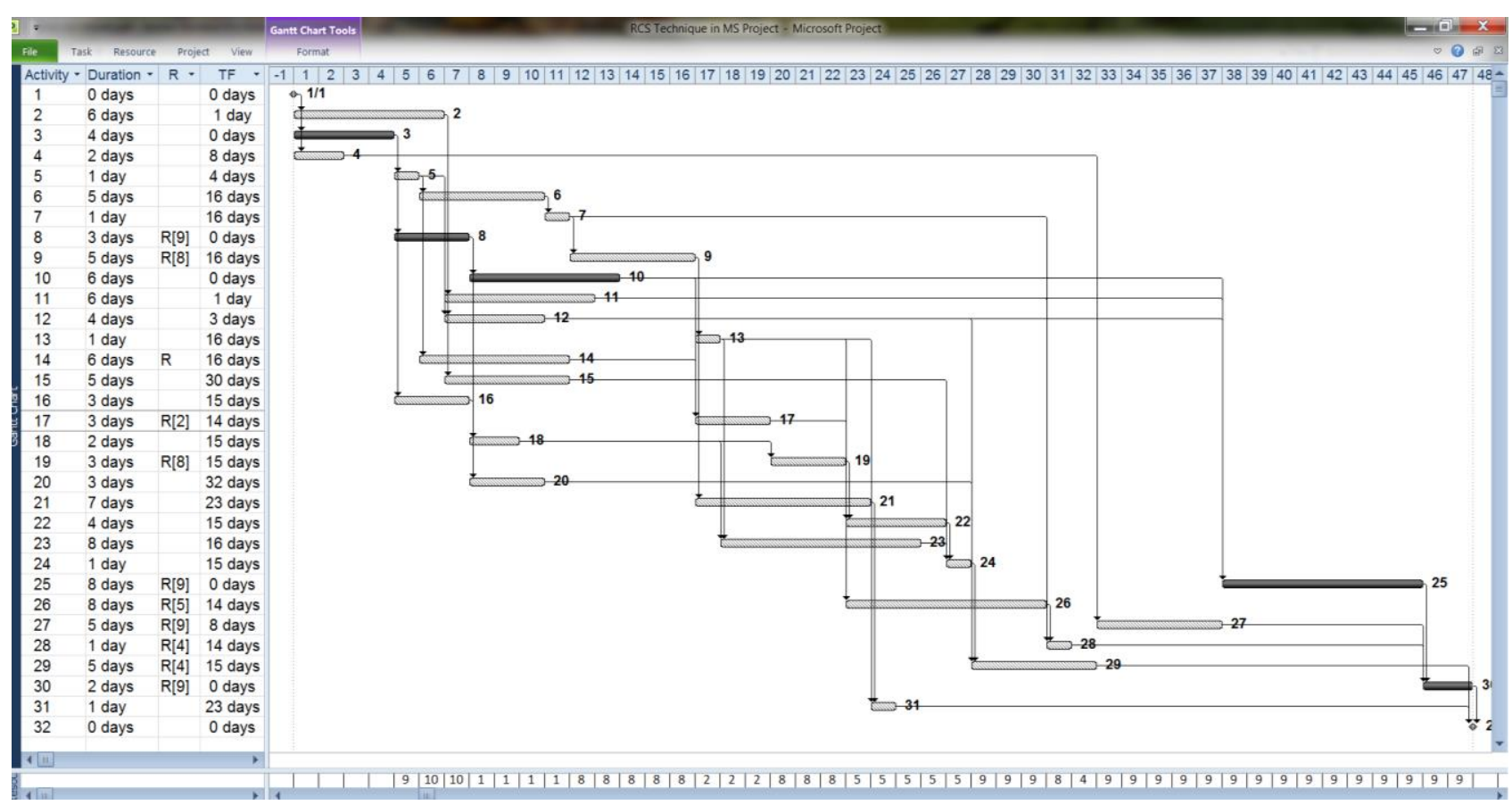

Fig.15 calculated schedule of case study network by RCS technique in MS Project 2010 


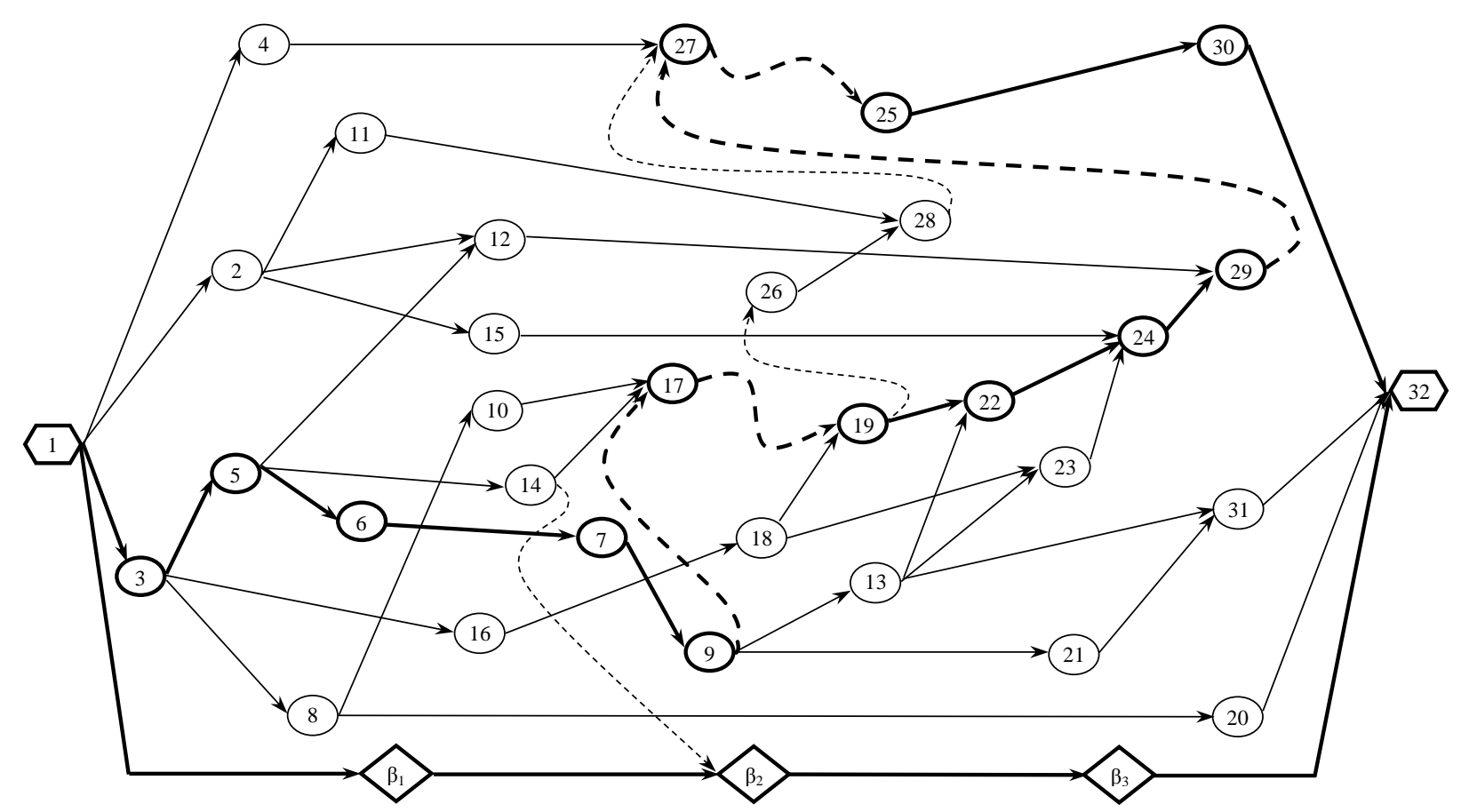

Fig.16 Schedule network of case study after application of enhanced RDCPM

To identify the resource dependencies in a project schedule under variable resource constraints in order to compute the correct floats and critical path, we improved the concept of RDCPM as an enhanced RDCPM in this study.

Work sequence in a resource constrained project schedule cannot be stable while the project is updated. Considering resource dependencies and establishing resource links between activities may generate a stable work sequence with updateable schedule. However, if the amount of available resources varies during the execution of a project, it may not be possible to identify resource dependency for some activities. Therefore, the enhanced RDCPM solved this problem by inserting dummy activities with allocating artificial resources into the schedule.

The application of enhanced RDCPM is performed to a complicate case study schedule taken

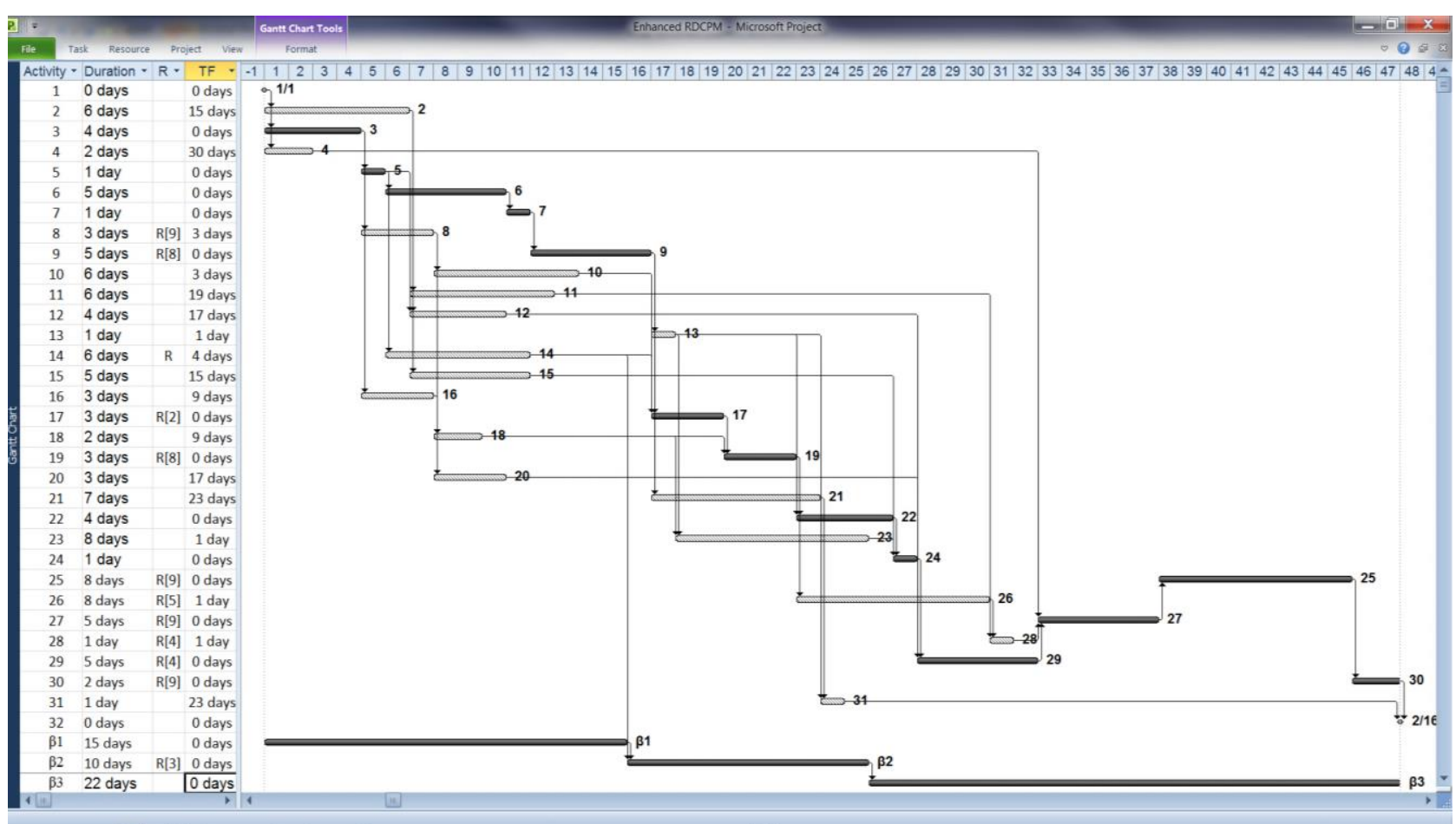

Fig.17 Calculated schedule of case study by enhanced RDCPM in MS Project 2010 
from the project scheduling problem library (PSPLIB). As observation on the results, the enhanced RDCPM provides reliable floats, the critical path, and stable project work sequences with an updateable schedule.

After the application of enhanced RDCPM, the schedule becomes as a CPM schedule that has a stable work sequence, and the resource limitation is satisfied. Hence, the schedule will be applicable to any kind of scheduling methods as well as to any project management software packages such as Primavera P6 and MS Project.

\section{References}

1) Ahuja, H. :Project management techniques in planning and controlling construction projects, Wiley, USA, 1984.

2) Kim, K. and De la Garza, J. M. : Phantom float, J. Constr. Eng. Manage., Vol. 129, No. 5, pp. 507-517, 2003.

3) Bowers, J. A. : Criticality in resource-constrained networks, J. Oper. Res. Soc., Vol. 46, No 1, pp. 80-91, 1995.

4) Wiest, J. D. : Some properties of schedules for large projects with limited resources, Oper. Res., Vol. 12, pp. 395-418, 1963.

5) Woodworth B M. and Shanahan S. : Identifying the critical sequence in a resource constrained project. Int. J. of Proj. Manage., Vol. 6, No. 2, pp. 89-96, 1988.

6) Kim, K. and De la Garza, J. M. : Evaluation of the resource constrained critical path method algorithms, J. Constr. Eng. Manage., Vol. 131, No. 5, pp. 522-532, 2005.

7) Lu, M. and Li, H. : Resource-activity critical-path method for construction planning, J. Constr. Eng. Manage., Vol. 129, No. 4, pp. 412-420, 2003.

8) Nisar, S. A., Yamamoto, K., and Suzuki, K. : Resource-dependent critical path method for identifying the critical path and the "real floats" in resource-constrained project scheduling, Construction Management, JSCE, vol. 69, No. 4, pp. I_97-I_107, 2013.

9) Nisar, S. A., Yamamoto, K., and Suzuki, K. : Scheduling model to identifying critical path and real total float under variable resource, $7^{\text {th }}$ International Conference on Project Management, ProMAC2013, 2013.

10) Kolsich, R. : Serial and parallel resource-constrained project scheduling methods revisited: Theory and computation, European Journal of Operational Research, vol. 90, pp. 320-333, 1996

11) Tam, P. W. M. and Dissanayake, P. B. G. : Construction project scheduling by ranked positional weight method, Can. J. Civ. Eng., Vol. 25, pp. 424-436, 1998.

12) Mubarak, S. : Construction project Scheduling and Control. $2^{\text {nd }}$ ed., John Wiley \& Sons, Canada, 2008.

13) Bashir. H. A. : Removal of redundant relationships in an AON project network for evaluating schedule complexity, $J$. Constr. Eng. Manage., Vol. 136, pp. 787-793, 2010.

14) Galloway, D. P. : Survey of the construction industry relative to the use of CPM scheduling for construction projects, J. Constr. Eng. Manage., Vol. 132, No. 7, pp. 697-711, 2006.

15) Kolisch, R. and A. Sprecher. : PSPLIB - A project scheduling library, European Journal of Operational Research, Vol. 96, pp. 205-216, 1996.

(Received October 7, 2013) 